\title{
Apuntes sobre una posible agenda de investigación para la didáctica de la
} física en Latinoamérica ${ }^{+}$

\section{Introducción}

Ha pasado ya un año entero desde que la pandemia de COVID-19 desatara una cascada de forzados, profundos y acelerados cambios en la enseñanza de la física en todos los niveles educativos (desde el básico hasta el superior), provocando disrupciones en las prácticas tradicionales del profesorado y suscitando nuevos problemas sobre los cuales reflexionar e investigar. Es un buen momento, entonces, para volver una vez más la mirada sobre nuestra disciplina - a la que voy a llamar con el nombre que encuentro técnicamente más correcto, didáctica de la física- e intentar identificar en ella estabilidades, avances, obstáculos, vacancias y desafíos. Tal es el propósito que avizora este editorial.

Los cambios de los que hablo, al haber hecho tambalear muchas de las "certezas" casi inamovibles que circulaban en torno a la enseñanza formal (iy presencial!) de la física, han arrojado nuevas luces sobre las problemáticas de investigación e innovación que compartimos dentro de nuestra comunidad académica. En este sentido, nos podemos preguntar críticamente hasta qué punto "el sistema educativo se ha renovado, para poder así hacerle frente a las nuevas demandas educativas" (HERRERO-VILLAREAL; ARGUEDASMATARRITA; GUTIÉRREZ-SOTO, 2020, p. 182) en el caso de la enseñanza de la física, signada por una impronta fuertemente conservadora. Con estas consideraciones en mente, me interesa examinar la cuestión de la oportunidad de plantear una actualización de la "agenda" esto es, del conjunto articulado de metas de mediano plazo- para la didáctica de la física, poniendo especial foco en nuestra región, Latinoamérica.

La didáctica de la física1 (“[pesquisa em] ensino de física”, en portugués; "physics education [research]", en inglés) es una disciplina -o, alternativamente, una subdisciplina dentro de la didáctica de las ciencias naturales/experimentales (ensino de ciências, science education $)^{2}$ - con un desarrollo histórico que ya podríamos considerar como relativamente largo (ver, por ejemplo, SALÉM, 2012). Hablo de una historia "relativamente larga" en el

\footnotetext{
${ }^{+}$Notes on a possible agenda for physics education research in Latin America

* Recebido: fevereiro de 2021. Aceito: fevereiro de 2021.

1 También llamada a veces "enseñanza de la física" o "educación en física".

2 Se la considera una subdisciplina que hace parte integral de la didáctica de las ciencias naturales en, por ejemplo, BORTOLETTO et al., 2007; GUERRA; MORA; GURGEL, 2020.
} 
sentido de que, a pesar de que 50 años resultan pocos en el marco mucho más amplio del desenvolvimiento de la ciencia moderna, son más que suficientes para que las primeras investigadoras e investigadores de didáctica de la física se hayan retirado ya, e incluso algunos de ellos, lamentablemente, nos hayan dejado. Es así que prácticamente no circula hoy en día, en nuestros encuentros académicos, "historia viva" - y con ella, memoria activa- de la constitución del campo. Ello torna necesario y urgente documentar nuestros orígenes y progreso a través de los testimonios de sus protagonistas, y ya hay algunos intentos en este sentido (e.g. NARDI, 2005a, 2005b) que se pueden recuperar como fuentes de apoyo pertinentes para mis argumentos.

A mitad de camino de su decurso histórico, sobre todo en los años 90, la didáctica de la física, por entonces una disciplina "emergente", dedicó una fracción de sus esfuerzos de pesquisa a preguntarse sobre sus fundamentos y su estatus epistemológico (de este estilo son los trabajos de TIBERGHIEN, 1985; NIEDDERER, 1999; ROSADO, 1999; GUISASOLA, 2005). Estas reflexiones metateóricas (“de segundo orden”) son otro material que orienta las consideraciones que hago en este texto.

Por otra parte, una contracara feliz de tener historia consolidada que me interesa recuperar en este editorial es que la didáctica de la física se ha vuelto "enseñable" (un claro rasgo de madurez epistemológica); es ahora una disciplina, asignatura o materia incluida en todos los cursos de formación docente para primaria y secundaria, con unos temas o tópicos ya más o menos establecidos y unos libros de texto que apoyan su enseñanza (por ejemplo, LOPES, 2004; CASTILBLANCO ABRIL; NARDI, 2014, en portugués; CAAMAÑO, 2011; KLEIN, 2012, en español; ARONS, 1997, en inglés; TOUSSAINT, 1996, en francés; KNOLL, 1978, en alemán). Esos textos me sirven también para caracterizar pasado, presente $\mathrm{y}$ futuro de nuestra disciplina.

Adicionalmente, a raíz de esta trayectoria no desdeñable de la que estoy hablando, la didáctica de la física va acumulando un contundente cuerpo de resultados empíricos y de desarrollos teóricos que lleva a la necesidad de que periódicamente se realicen revisiones (reviews) y meta-análisis ${ }^{3}$. Recupero algunos de esos trabajos -como explicaré más abajo, aquellos de corte más general y sinóptico y con mayor cantidad de categorías teóricas organizadoras- a fin de enriquecer las proposiciones y sugerencias que hago en este escrito.

A todo el anterior corpus de referencia sumo por último algunos documentos de trabajo, producidos - generalmente por encargo- al interior de asociaciones de didactas de la física con la intención de servir de insumos para la discusión sobre el rumbo de la disciplina ${ }^{4}$.

\footnotetext{
3 En este parágrafo de sistematizaciones meta-analíticas se incluirían los "handbooks" -compilaciones extensivas del estado del arte de la disciplina de las que participan reconocidos expertos. En esa dirección, muy recientemente se ha lanzado un proyecto internacional ambicioso, el International Handbook of Physics Education Research (http://ijpce.org/index.php/IHPER/index).

${ }^{4}$ En este ítem destaca PER-Central (https://www.compadre.org/per/), un notable sitio web sobre el surgimiento, estado actual y rasgos caracterizadores de la didáctica de la física en los Estados Unidos, con variada documentación informativa, analítica y crítica.
} 
Dicho todo esto, advierto a las lectoras y lectores que el presente texto, como corresponde a todo editorial, no es un recorrido sistemático por la literatura didáctica, sino una síntesis valorativa personal y una nota de opinión. Por tanto, cuando -en las dos últimas secciones- identifique temas y problemas que creo nodales para la didáctica de la física del presente y del futuro cercano (al menos en nuestra región), omitiré hacer citas a grupos y autores concretos que los representen, a fin de evitar el riesgo de que este trabajo resulte sesgado o incompleto. En mi editorial solo se exponen "algunos elementos que permiten situar la evolución de las actividades de investigación en didáctica de la física, no es pues aquí cuestión de ser exhaustivos” (TIBERGHIEN, 1985, p. 71, la traducción es mía).

\section{La relativa "autonomía" de la didáctica de la física}

La didáctica de la física como como campo científico enlaza, a nivel internacional, dos tradiciones de trabajo bien distinguibles que se remontan a sus primeros tiempos “disciplinados" en los años 70. Creo que resulta importante reconocerlas para saber en qué coordenadas nos movemos como comunidad académica. En una de esas tradiciones se busca "unidad en la diversidad": se pone énfasis en la compartición de marcos teóricos y metodológicos con las didácticas de la química, biología, geología, astronomía, etc. En la otra, en cambio, se considera que la didáctica de la física es relativamente autónoma de la didáctica de las ciencias naturales y de la investigación educativa y se ubica más cerca de la propia física: hecha desde los departamentos universitarios de esta disciplina (ver al respecto HERON; MELTZER, 2005).

Podemos vincular la primera tradición, que llamaríamos "unionista", a la asociación europea ESERA (European Science Education Research Association), a su contraparte estadounidense NARST (National Association of Research in Science Teaching), a las revistas International Journal of Science Education (Reino Unido) y Enseñanza de las Ciencias (España) y al congreso brasileño ENPEC (Encontro Nacional de Pesquisa em Educação em Ciências). Esta tradición inserta los estudios científicos sobre la enseñanza y el aprendizaje de la física (realizados sobre todo en los niveles primario y secundario) en el panorama más amplio de la preocupación por la calidad de la educación en ciencias; por tanto, considera que la didáctica de la física sería una "rama" o "capítulo" de la didáctica de las ciencias naturales, de la cual tomaría sus grandes problemas, sus marcos referenciales y autores "citables", y sus orientaciones metodológicas. Hay debajo de este acercamiento la tesis de que

las herramientas conceptuales elaboradas por los investigadores [en la didáctica de una disciplina] para definir su problemática, especificar su metodología y formular sus resultados apuntan a una cierta generalidad transdisciplinar (VIENNOT, 1997, p. 76, la traducción es mía). 
La segunda tradición, que calificaremos de "secesionista", se vincula por su parte más a la red de investigación europea GIREP (Groupe International de Recherche sur l'Enseignement de la Physique), a la revista estadounidense American Journal of Physics y a físicos de ese país pioneros en la pesquisa sobre enseñanza, como Lillian McDermott, Robert Karplus y David Hestenes 5 , a la International Commission on Physics Education de la IUPAP y, en nuestra región, a la revista Latin-American Journal of Physics Education y a los congresos EPEF (Encontro de Pesquisa em Ensino de Física) de Brasil y SIEF (Simposio de Investigación en Educación en Física) de Argentina. Esta tradición pone más énfasis en la enseñanza preuniversitaria y universitaria y en tópicos de física especializados, incluso muy sofisticados. Así, va más allá del tratamiento sistemático de las cuestiones pedagógicas tradicionales del desarrollo curricular y el diseño de la enseñanza escolar y se configura como "investigación enfocada en lo que sucede cuando los estudiantes se esfuerzan por captar y usar los conceptos de la física" (BEICHNER, 2009, p. 3, la traducción es mía).

Desde el punto de vista teórico, esta tradición secesionista a todas luces conserva, mucho más que la unionista, un fuerte sesgo "psicologista", tomando muchas de sus referencias conceptuales del campo de la cognición y el aprendizaje. Desde el punto de vista metodológico, se podría afirmar que tiene una marcada trayectoria de investigación cuantitativa, con un uso extensivo de técnicas estadísticas, que es herencia de la formación "física" de sus cultivadores (ver HERON; MELTZER, 2005, p. 390).

Es crucial señalar que las dos tradiciones de didáctica de la física que acabo de describir no son para nada disjuntas; antes bien, pueden considerarse dos enfoques o "estilos" complementarios que conviven armónicamente. De hecho, la inmensa mayoría de los didactas de la física transitan ambos estilos y desarrollan sus ideas y presentan sus resultados en los foros que asocié a las dos tradiciones.

Por último, resulta interesante notar que, en muchos de los trabajos científicos (en sus distintos formatos) que estoy tomando para fundamentar mi estado del arte, las investigaciones de didáctica de la física reportadas aparecen clasificadas bajo descriptores generales propios del campo pedagógico (a saber: enseñanza secundaria, formación del profesorado, evaluación, aprendizaje, discurso en el aula, etc.), y esto, sorprendentemente, se constata para ambas tradiciones. Hay pocos trabajos en una $\mathrm{u}$ otra que hacen emerger categorías taxonómicas más cercanas a los problemas propios de la enseñanza de la física en los distintos escenarios educativos con el fin de sistematizar el estado de desarrollo de nuestro campo; me referiré a esos trabajos en la próxima sección.

\footnotetext{
5 En la mencionada PER-Central se incluye un muy interesante "árbol genealógico" de nuestra disciplina en los Estados Unidos (http://www.compadre.org/per/wiki/PERFamilyTrees.jpg). El árbol se construye a partir de aquellos investigadores que la comunidad considera fundadores del campo y organiza el mapa de sus principales discípulos.
} 


\section{Líneas de investigación de la didáctica de la física}

Para que se entienda bien el propósito de esta sección, me resulta necesario fijar con un poco de cuidado el alcance de la expresión "línea de investigación". En la didáctica de la física existe una enorme variedad de temas, objetos, problemas y preguntas que se investigan: el campo está explosionado ${ }^{6}$. Para que un conjunto bien articulado de esos temas y problemas constituya una "línea" es necesario que haya continuidad en el tiempo, masa crítica de investigadores y de producciones y unos referenciales teórico-metodológicos reconocibles y estabilizados. Por tanto, presentar un listado de líneas "vigentes" en la didáctica de la física implica inevitablemente realizar un recorte de la diversidad existente, decantándose por los modos dominantes de investigar. Esta aclaración por supuesto impone límites sensatos al estado del arte que hago en esta sección, pero a la vez abre una cuestión problemática para la siguiente: la de las relaciones entre centro y periferia en el universo académico, en el sentido de si es posible pensar en líneas de investigación "reconocidas" por el establishment científico central cuya producción se documente en portugués o en español y emane de los países latinoamericanos, etiquetados como "en vías de desarrollo".

Como ya dije, existe un número no desdeñable de reseñas sobre el estado del arte de la didáctica de la física (solo para citar algunas pocas, a modo de ilustración: BORTOLETTO et al., 2007; REZENDE; OSTERMANN; FERRAZ, 2009, en portugués; GUISASOLA et al., 2004; GARCÍA CARMONA, 2009, en español; BEICHNER, 2009; CUMMINGS, 2011; DOCKTOR; MESTRE, 2014; MELTZER; OTERO, 2015, en inglés). Estas reseñas se proponen "mapear" la producción investigativa del campo emplazándola en distintas categorías. Adicionalmente, las revistas y congresos académicos de nuestra disciplina también organizan la investigación e innovación mediante "ejes" o "hilos" temáticos. Según pude observar en ambos casos, las etiquetas para describir qué investigamos los didactas de la física son la mayor parte de las veces demasiado generales, y en este sentido me sirven solo parcialmente para el objetivo de este editorial.

A partir de todo ese material, en el que existen - hasta donde pude encontrar- unas pocas reseñas que proponen constructos más específicos para la tarea, haré aquí un intento de señalar líneas de trabajo "estabilizadas" en la didáctica de la física y de apuntar algunas otras que parecen estar surgiendo. Sumaré a ello algún apunte final sobre temas difíciles de incluir en las grandes líneas, que por ello pueden quedar poco visibles, pero que interesa tener en cuenta para armar un cuadro más completo e intuir nuevos rumbos de trabajo.

La línea de investigación más citada en las revisiones de las diferentes décadas es la del aprendizaje o comprensión de los conceptos físicos (nombrada como "conceptual understanding" en la literatura en inglés) (ver McDERMOTT; REDISH, 1999; BEICHNER, 2009; DOCKTOR; MESTRE, 2014). Esta línea hunde sus raíces en los trabajos fundacionales

\footnotetext{
6 De hecho, la famosa investigadora estadounidense en didáctica de la física Paula Heron -junto con su colega David Meltzer- planteaba, en un editorial para el American Journal of Physics (HERON; MELTZER, 2005), que esa explosión constituía un problema para el desarrollo del campo a inicios del siglo XXI.
} 
de la didáctica de la física sobre "concepciones alternativas" (en sus diferentes denominaciones), enseguida da paso al marco del cambio conceptual (saludado por la comunidad como la primera "teoría" propia) y luego, atravesando la etapa de incorporación del constructo de modelos mentales, evoluciona hacia el empleo de aportaciones cognitivas actualizadas y sofisticadas para estudiar con cierto detalle los procesos de aprendizaje de la física en el estudiantado. Esta es una línea que, reinventándose continuamente por medio de la cooptación de nuevas perspectivas teóricas, sigue muy sana en nuestros días.

En solapamiento parcial con la línea anterior se reconoce un conjunto grande de trabajos sobre los aspectos "epistémicos" del aprendizaje de la física, que tocan tópicos tales como el uso de las analogías, la naturaleza del razonamiento de sentido común, la incorporación de reglas lógicas, las creencias epistemológicas, los problemas en torno al clásico "método científico" y la modelización física7. Se podría incluir aquí también el foco de trabajo sobre "la matemática en la física", que, sin ser mayoritario, acumula algunos logros importantes.

Se observa en las reseñas un intento de integrar esta línea con la anterior en un espacio común más amplio; para la integración se utiliza casi siempre el hiperónimo de "cognición en física". Ese espacio más abarcativo agrupa, sin lugar a dudas, el grueso de la producción en didáctica de la física desde sus inicios hasta comenzado el siglo XXI.

Por otra parte, tanto en el mundo anglosajón como en Iberoamérica siempre se pone en valor la línea sobre resolución de problemas, que en general aparece "maridada" con la investigación en torno a los trabajos prácticos y al laboratorio de física. Esta línea resulta fundamental para nuestra concepción de las posibilidades y límites de la física escolar y ha recibido un indisimulable sacudón en tiempos de pandemia con la pérdida de la presencialidad e incluso, en bastantes casos, de la simultaneidad en la enseñanza. En los últimos años, esta línea explora, en intersección con la de "nuevas tecnologías", tres modalidades alternativas de laboratorio: asistidos por ordenador, virtuales y remotos.

Cabe anotar aquí que, en algunas reseñas a las que he accedido, se habla de una línea paralela a la anterior, tematizada como el aprendizaje en el aula versus el aprendizaje en el laboratorio (por ejemplo, McDERMOTT; REDISH, 1999, p. 763-764); tal línea se apoya en una oposición retórica frontal entre las denominadas "metodologías activas" (un tópico muy de moda hoy en día) y las clases expositivas, o "lecciones" del maestro.

Otra línea que se reporta como presente a lo largo de toda la historia de la didáctica de la física es la de la integración de las tecnologías digitales en la enseñanza, con sus altibajos correlacionados con la situación política y económica de cada época y registrando importantes saltos adelante concomitantes con las pequeñas o grandes revoluciones que se

\footnotetext{
7 En opinión de muchos investigadores en la didáctica de las ciencias naturales, entre los que me cuento, "modelos y modelización" constituiría una línea de pesquisa separada por derecho propio, debido al énfasis que se viene poniendo en la naturaleza de los modelos como constructos teóricos de la ciencia. En la didáctica de la física, en cambio, la mayoría de las veces el foco está puesto en los procesos "internos" de representación de los fenómenos, y así la línea se diluye en el área más amplia a la que se nombra como "cognición en física”.
} 
fueron dando en el campo tecnológico en el último medio siglo. En esta línea, el uso de simulaciones computacionales en la física escolar viene ganando terreno, así como las reflexiones en torno al rol que pueden jugar las redes sociales en un aprendizaje más significativo.

También es recurrente la mención a una línea más nueva en torno a las creencias y actitudes sobre la física, su naturaleza, su aprendizaje y su enseñanza, primero enfocada en el estudiantado y luego ampliada al profesorado en formación y en actividad. Esta línea se encuentra con la de "cognición en física" en su interés por las nociones teóricas de metacognición y autorregulación.

Por su parte, desde mediados de los años 90 tiene gravitación la línea de investigación que utiliza insumos teóricos de la epistemología (o filosofía), la historia y la sociología de la ciencia para la mejora de las clases de física. Esta línea, más bien minoritaria, fue tradicionalmente "auxiliar" de la del aprendizaje de conceptos, pues proveía de análisis histórico-epistemológicos de las distintas teorías físicas que nos permitían a los investigadores prever obstáculos de comprensión. Luego se amplió notablemente para indagar sobre lo que se ha dado en llamar "naturaleza de la física": la enseñanza explícita (a estudiantes y profesores) de ideas metateóricas incluidas en los programas de física escolar o universitaria.

De acuerdo con mi visión de la didáctica de la física, esta línea de trabajo en torno a los aportes metacientíficos a la educación en física está ricamente desarrollada en Latinoamérica, probablemente debido a la historia de las instituciones (Escuelas Normales, Institutos Superiores, etc.) en las que se desplegó tradicionalmente la formación del profesorado en nuestra región.

Por otra parte, llama la atención que, a pesar de que Jay Lemke -quien acuñó la muy sugerente idea de "hablar ciencias" (LEMKE, 1990)- proviene del campo de la enseñanza de la física, la línea que agrupa el estudio del lenguaje en las clases, las competencias lingüísticas, la argumentación científica escolar y las narrativas es pocas veces mencionada en las reseñas. Esto supone una anomalía de categorización, dado que, por un lado, resulta forzado subsumir el "hablar física" en las demás líneas que ya he presentado y, por otro, pierde personalidad si se lo ubica en las que rescataré a continuación, que son más generalistas.

Por último, las revisiones y estados del arte reconocen la emergencia mucho más reciente de una línea de trabajo, caracterizada de manera vaga y con límites ciertamente difusos, sobre aspectos "sociales" de la enseñanza de la física, que se enfoca en los ambientes de aprendizaje, la motivación, la interacción, la colaboración entre estudiantes y la percepción de uno mismo y de los otros, y (aparentemente) incluye cuestiones de género, minorías, migrantes, capital cultural, uso de la lengua materna y de segundas lenguas, etc.

De manera transversal a esta forma muy específica de organizar los tópicos de investigación, es común utilizar también categorías educativas tradicionales -currículo, enseñanza, evaluación, desarrollo de materiales didácticos y formación inicial y continuada 
del profesorado-, especificándolas con el descriptor "de (la) física". En este conjunto de líneas se ubican tópicos tales como: finalidades de la educación en física y perspectiva CTS; "conocimiento pedagógico del contenido" del profesorado; investigación sobre los libros de texto didácticos; "gamificación" de la enseñanza; y organización de los currículos y programas por "competencias".

También podemos situar bajo este paraguas un tópico que tiene mucha fuerza en las comunidades de investigación europea continental y latinoamericana, la investigación en torno al planeamiento, puesta marcha y evaluación de unidades (o secuencias) didácticas de física. El enfoque tradicional para este tópico fue la evaluación "pre/post"; ahora se ha enriquecido metodológicamente con la perspectiva de la investigación basada en el diseño ("pesquisa baseada em design", en portugués; “design-based research”, en inglés).

Para cerrar el mapa de líneas que he bosquejado, me parece ineludible señalar que las reseñas del panorama de investigación internacional, fuertemente teñidas por el énfasis psicologista hegemónico puesto en la cognición, el aprendizaje y el estudiantado, invisibilizan lo que Manchón-Gordón y García Carmona (2018, p. 128) llaman "investigaciones sobre análisis de la práctica docente en el aula de física", que sí ocupan un lugar relevante en las comunidades con gran tradición pedagógica, como las del norte de Europa (Alemania, Suecia, Países Bajos) o Iberoamérica.

Ahora bien, párrafo aparte merecen aquellos temas que los didactas de la física reconocemos como investigables e investigados, pero que -debido a que son recientes y en algún punto "periféricos"- se resisten a ser clasificados en el sistema de líneas "ortodoxas". Aquí enumeraría, sin pretensión de barrer todo el arco de tópicos e inspirándome en los programas de los últimos EPEF y SIEF: la participación de las mujeres en la historia "oficial" de la física y los "saberes físicos de las mujeres" (utilizando la expresión sugerida por la investigadora catalana Núria Solsona [2015]); la enseñanza de la física en contextos multiculturales y multilingües; el uso de "historias de la física" en la enseñanza; la indagación "modelizadora" como estrategia metodológica coherente con la naturaleza de la física; la educación en física en contextos no formales o informales y su interacción con la divulgación y la comprensión pública de la ciencia; el lugar de la física en las propuestas de integración disciplinar, y en particular en el enfoque STE(A)M; la multimodalidad, o uso de múltiples recursos semióticos coordinados en clase; la introducción de la "mirada física" en la educación en edades tempranas (0-6 años); la enseñanza de la física para poblaciones con necesidades educativas especiales; y la contribución de la física a la formación de ciudadanía, pensamiento crítico, toma de decisiones informadas y participación en asuntos sociocientíficos.

Estos y otros tópicos innovadores que se escapan por ahora de la clasificación en líneas en cierta manera perfilan la agenda de investigación de la didáctica de la física para la próxima década. Tendremos que seguirles la pista en los eventos y publicaciones. 


\section{Una agenda latinoamericana para la didáctica de la física}

En esta última sección quiero discutir las posibilidades de existencia de lo que en algunos trabajos anteriores he venido llamando una didáctica de la física en Latinoaméríca, de Latinoamérica y para Latinoamérica (ADÚRIZ-BRAVO, 2007, 2013).

Que hay abundante y contundente producción identificable como didáctica de la física (con los matices de nomenclatura que he señalado más arriba) en Latinoamérica parece fácil de constatar; basta con sobrevolar las revisiones que ya he citado y añadir a ellas compilaciones locales o regionales multiautoradas sobre el tema (por ejemplo: VALDÉS et al., 2002; GARCIA et al., 2010; SANTOS; STIPCICH, 2010) y reportes de los congresos que se realizan en nuestra región.

En efecto, si echamos una rápida ojeada a los libros de resúmenes, actas y "proceedings" de EPEF y SIEF (eventos a los que podemos sumar el SNEF-Simpósio Nacional de Ensino de Física en Brasil y la REF-Reunión de Educación en Física en Argentina, en los que se presenta innovación educativa fundamentada o indagación en las aulas), advertimos sin dificultades la coherencia y la continuidad de la producción en didáctica de la física: se reconocen nombres destacados, se identifican grupos y líneas de trabajo muy estables y se advierte regularidad en la defensa de tesis de posgrado en el campo. Si a la masa crítica de recursos humanos relevados sumamos al menos las comunidades más pequeñas de didactas de la física que hay en México, Colombia, Chile y Uruguay (comunidades que también tienen sus propios encuentros), podemos comprobar que se supera holgadamente el centenar de personas a las que se puede considerar investigadores latinoamericanos activos en el campo. Se trata, por tanto, de un colegio profesional nada desdeñable.

Ahora bien, en ese mismo recorrido en diagonal también se puede constatar que buena parte de la producción académica disponible retoma los temas de la agenda internacional instalada y cita las producciones de los países tenidos como hegemónicos; en este sentido, resulta fácil ubicarla en el panorama que esbocé en la sección anterior. Sin embargo, creo que de todas maneras estamos en condiciones de hablar de una didáctica de la física de Latinoamérica, en el sentido de que una parte representativa de las producciones locales es altamente original y supone avances teóricos, conclusiones empíricas o aportes metodológicos que a veces logran impactar en el escenario global de discusiones.

Por ejemplo, desde el punto de vista de los tópicos de investigación, es posible reconocer trabajos sobre: aspectos socioculturales de la física y su enseñanza en nuestros países, signados por un pasado colonial; saberes de los pueblos originarios en torno al mundo físico y su interacción con la física "europeizante"; experimentos y textos históricos (extranjeros y locales) que se pueden usar en las aulas de física y en la formación docente; renovación de las explicaciones de aula y de las prácticas de laboratorio en contextos con múltiples constreñimientos (sobrepoblación de los salones, falta de infraestructura y equipamiento adecuados, pobreza o violencia estructurales, profesorado a cargo con poca o 
nula formación docente); problemáticas de enseñar física a personas con distintos tipos de discapacidades; y otras varias temáticas más que están todavía relativamente poco desarrolladas a nivel mundial, en las cuales esta contribución latinoamericana es potencialmente relevante.

Desde el punto de vista de la fundamentación teórica, por su parte, hay investigadores de didáctica de la física en Latinoamérica que se especializan en las ideas de epistemólogos (filósofos de la ciencia) como Gaston Bachelard, Ronald Giere o Bruno Latour, lingüistas como Mijaíl Bajtín, Chaïm Perelman o Christian Plantin y educadores como Lev Vygotski o Paulo Freire, autores que no están tan transitados en el campo a nivel internacional. Hay en nuestro continente, por tanto, experticia de primer nivel que puede aportar al avance de la disciplina en su conjunto.

También desde nuestra región se ha ampliado la literatura de la didáctica de la física de manera original y sustantiva, trayendo contribuciones teórico-metodológicas de psicólogos y especialistas en ciencia cognitiva y neurociencia, historiadores de la ciencia, autores del campo de los estudios sociales de la ciencia y la tecnología, teóricos de la didáctica de la matemática "antropológica" de tradición francesa, y académicos que trabajan desde las perspectivas crítica, decolonial, feminista, de la complejidad, queer, etc. En mi opinión se constata que, en no pocos casos, Latinoamérica aporta variedad y calidad en los fundamentos teóricos de sus investigaciones didácticas.

Queda, por último, hacernos la pregunta sobre la constitución de una didáctica de la física para Latinoamérica, en el sentido de poder poner en marcha, de manera planificada y sostenida, una agenda de investigaciones sobre nuestra realidad que, conociéndola a fondo, logren tener impacto en su transformación. Creo que en esta dirección todavía hay muy poco hecho, pero tenemos, por lo que dije anteriormente, todos los "ingredientes" a la mano para avanzar en la tarea en los próximos años:

1. Una satisfactoria diagnosis de la educación y de la enseñanza de las ciencias en nuestra región que, aceptando la universalidad de muchos problemas didácticos, ya comienza a conocer bien aquellos que son específicos de Latinoamérica y a advertir los matices que toman en los distintos países que constituyen la región.

2. Unos temas de investigación y unos marcos teóricos originales y potentes como los que reconocí más arriba.

3. Una formulación de los problemas de investigación con cada vez mayor relevancia social, que no esquiva considerar seriamente los detalles del contexto de trabajo y asume las condiciones de posibilidad de una educación en física de nuestros niños y niñas, adolescentes y jóvenes dirigida a la formación de ciudadanía.

4. Un compromiso inclaudicable de los didactas de la física latinoamericanos con valores humanistas como la democracia, la equidad, la inclusión, el respeto por la diversidad en todas sus formas y la justicia social, en pos de la transformación de nuestra región. 
5. Recurso humano joven con formación de alta calidad, en números aceptables como para constituir comunidades académicas compactas en los distintos países y garantizar la continuidad y expansión de la disciplina.

6. Redes de trabajo de escala regional que son capaces de vincular genuinamente a los didactas de la física latinoamericanos, ayudadas por la enorme extensión de nuestras dos lenguas vehiculares (portugués y español, con más de 700 millones de hablantes) y por la mejora de la conectividad física y virtual entre nuestros países. Y, lo que es más importante, una fuerte voluntad de establecer lazos de colaboración, que se ve reflejada patentemente en la presencia de investigadores de varios de nuestros países en los eventos académicos que se realizan periódicamente en la región.

7. Media docena de revistas de didáctica de la física con trayectoria, prestigio e impacto, que funcionan como órganos de difusión de la producción para Iberoamérica y el resto del mundo.

Con todo lo discutido, creo que las claves para poder constituir una didáctica de la física en, de y para el continente pasarían por los siguientes carriles: adoptar de lleno una franca perspectiva sociocultural de la física cuidadosamente adecuada a las realidades de nuestra región, que nos permita dar nuevas respuestas, axiológicamente consistentes, a la pregunta de para qué enseñar la física -con todo lo que ella connota- en las escuelas de Latinoamérica; reformar a fondo programas y materiales liberándonos del lastre de una concepción heredada de física académica y propedéutica, que siempre mira "hacia afuera"; ser audaces en la construcción de innovadores sistemas teóricos locales, buscando creciente independencia de los marcos foráneos; plantear acercamientos metodológicos robustos que formulen y ataquen problemas didácticos "a pie de aula" y con la participación de profesores; y orquestar unos mecanismos de formación docente que pongan en el centro la autonomía y la profesionalidad de quienes irán a las aulas de física.

Falta aún mucho por hacer, pero la revisión que he realizado del estado del arte en nuestro campo me convence de que estamos en la dirección correcta hacia lograr poner una impronta única $-\mathrm{y}$ reconocible en todo el planeta- a la didáctica de la física latinoamericana.

\section{Agradecimiento}

Agradezco a las Profesoras Andreia Guerra e Irene Arriassecq por su asistencia en la búsqueda de fuentes bibliográficas regionales para este texto.

\section{Referencias bibliográficas}

ADÚRIZ-BRAVO, A. Didáctica de las ciencias naturales y didáctica de la física: ¿De dónde venimos, dónde estamos y hacia dónde vamos? Conferencia plenaria. REUNIÓN DE EDUCACIÓN EN LA FÍSICA, XV, 2007, Villa de Merlo, Argentina. 
ADÚRIZ-BRAVO, A. La evolución de la investigación en enseñanza de las ciencias en Iberoamérica. Conferencia plenaria. WEF@-1ER WORKSHOP ENSEÑANZA DE LA FÍSICA: LOS DESAFÍOS DE LA INVESTIGACIÓN EDUCATIVA Y LA FORMACIÓN DOCENTE, 2013, Tandil, Argentina.

ARONS, A. B. Teaching introductory physics. New York: Wiley, 1997.

BEICHNER, R. An introduction to physics education research. In: Getting started in PER, v. 2, 2009. Disponible en:

$<$ http://www.compadre.org/Repository/document/ServeFile.cfm?ID=8806\&DocID=1147>.

Acceso en: 26 ene. 2021.

BORTOLETTO, A. et al. Pesquisa em ensino de física (2000-2007): áreas temáticas em eventos e revistas nacionais. In: ENCONTRO NACIONAL DE PESQUISA EM EDUCAÇÃO EM CIÊNCIAS, 6, 2007, Florianópolis. Atas... Florianópolis: ABRAPEC, 2007. Disponible en <http://gpeqsc.com.br/guaipira/artigos/2007_6_ENPEC.31.pdf >. Acceso en: 26 ene. 2021.

CAAMAÑO, A. (Coord.). Didáctica de la física y química. Barcelona: Graó, 2011.

CASTIBLANCO ABRIL, O. L.; NARDI, R. Didática da física. 1. ed. São Paulo: Cultura Acadêmica, 2014. Disponible en: <http://hdl.handle.net/11449/126216>.

CUMMINGS, K. A developmental history of physics education research. In: SECOND COMMITTEE MEETING ON THE STATUS, CONTRIBUTIONS, AND FUTURE DIRECTIONS OF DISCIPLINE-BASED EDUCATION RESEARCH, 2011. Disponible en: $<$ https://sites.nationalacademies.org/cs/groups/dbassesite/documents/webpage/dbasse_072580 .pdf $>$. Acceso en: 26 ene. 2021.

DOCKTOR, J. L.; MESTRE, J. P. Synthesis of discipline-based education research in physics. Physical Review Special Topics: Physics Education Research, v. 10, n. 2, p. 020119-1-020119-58, jul.-dec. 2014.

GARCIA, N. M. D. et al. (Orgs.). A pesquisa em ensino de física e a sala de aula: articulações necessárias. São Paulo: Livraria da Física, 2010. 352 p.

GARCÍA-CARMONA, A. Investigación en didáctica de la física: tendencias actuales e incidencia en la formación del profesorado. Latin-American Journal of Physics Education, v. 3, n. 2, may. 2009. 
GUERRA, A.; MOURA, C. B.; GURGEL, I. Sobre educação em ciências, rupturas e futuros (im)possíveis. Caderno Brasileiro de Ensino de Física, v. 37, n. 3, p. 1010-1019, dic. 2020.

GUISASOLA, J. La investigación en la enseñanza de la física: de la anécdota a la producción de conocimiento científicamente fundamentado. Investigações em Ensino de Ciências, v. 10, n. 1, p. 103-127, 2005.

GUISASOLA, J. et al. ¿Puede ayudar la investigación en enseñanza de la física a mejorar su docencia en la universidad? Revista Brasileira de Ensino de Física, São Paulo, v. 26, n. 3, p. 197-202, 2004. Disponible en:

$<$ http://www.scielo.br/scielo.php?script=sci_arttext\&pid=S1806-11172004000300002\&lng $=$ en\&nrm=iso $>$. Acceso en: 26 ene. 2021.

HERON, P. R. L.; MELTZER, D. E. The future of physics education research: Intellectual challenges and practical concerns. American Journal of Physics, v. 73, n. 5, p. 390-394, 2005. Disponible en: <https://doi.org/10.1119/1.1858480>. Acceso en: 26 ene. 2021.

HERRERO-VILLAREAL, D.; ARGUEDAS-MATARRITA, C.; GUTIÉRREZ-SOTO, E. Laboratorios remotos: recursos educativos para la experimentación a distancia en tiempos de pandemia desde la percepción de estudiantes. Revista de Enseñanza de la Física, v. 32, n. extra, p. 181-189, 2020.

KLEIN, G. Didáctica de la física, 2012. Disponible en:

$<$ http://www.anep.edu.uy/ipafisica/document/material/cuarto/2008/didac_3/did_fis.pdf $>$. Acceso en: 26 ene. 2021.

KNOLL, K. Didaktik der Physik: Theorie und Praxis des Physikunterrichts in der Sekundarstufe 1. München: Ehrenwirth, 1978.

LEMKE, J. L. Talking science: Language, learning, and values. Norwood, NJ: Ablex, 1990. $261 \mathrm{p}$.

LOPES, J. B. Aprender e ensinar física. Lisboa: Fundação Calouste Gulbenkian/Fundação para a Ciência e a Tecnologia, 2004. 430 p.

MAGAZÙ, S. (Ed.). New trends in physics education research. New York: Nova Science Publishers, 2018. 318 p. 
MANCHÓN-GORDÓN, A. F.; GARCÍA-CARMONA, A. ¿Qué investigación didáctica en el aula de física se publica en España? Una revisión crítica de la última década para el caso de educación secundaria. Enseñanza de las Ciencias, v. 36, n. 2, p. 125-141, jun. 2018.

McDERMOTT, L. C.; REDISH, E. F. Resource Letter: PER-1: Physics Education Research. American Journal of Physics, v. 67, n. 9, p. 755-767, 1999. Disponible en:

$<$ https://doi.org/10.1119/1.19122>. Acceso en: 26 ene. 2012.

MELTZER, D. E.; OTERO, V. K. A brief history of physics education in the United States. American Journal of Physics, v. 83, n. 5, p. 447, 2015. Disponible en:

$<$ https://doi.org/10.1119/1.4902397>. Acceso en: 26 ene. 2021.

NARDI, R. Memórias da educação em ciências no Brasil: a pesquisa em ensino de física. Investigações em Ensino de Ciências, Porto Alegre-RS, v. 10, n. 1, 2005a. Disponible en: $<$ http://www.if.ufrgs.br/public/ensino/vol10/n1/v10_n1_a4.htm>. Acceso em: 26 ene. 2021.

NARDI, R. A área de ensino de Ciências no Brasil: fatores que determinaram sua constituição e suas características segundo pesquisadores brasileiros. 2005b. 169 p. Tese [Livre Docência] - Faculdade de Ciências, Universidade Estadual Paulista, Bauru.

NIEDDERER, H. Recherche et développement en didactique de la physique à l'université: résultats et tendances (Research and development in didactics of the physics to the University: Results and tendencies). Didaskalia, n. 14, p. 95-113, 1999.

REZENDE, F.; OSTERMANN, F.; FERRAZ, G. Ensino-aprendizagem de física no nível médio: o estado da arte da produção acadêmica no século XXI. Revista Brasileira de Ensino de Física, São Paulo, v. 31, n. 1, p. 1402.1-1402.8, abr. 2009. Disponible em:

$<$ http://www.scielo.br/scielo.php?script=sci_arttext\&pid=S1806-11172009000100008\&lng $=$ en\&nrm=iso $>$. Acceso en: 26 ene. 2021.

ROSADO, L. Didáctica de la física y sus nuevas tendencias: Manual de 1999. Madrid: UNED, 1999.

SALÉM, S. Perfil, evolução e perspectivas da pesquisa em ensino de física no Brasil. 2012. 385 p. Tese (Doutorado em Ciências) - Instituto de Física, USP, São Paulo.

SANTOS, G.; STIPCICH, S. (Comps.). Tecnología educativa y conceptualización en física: Estudio sobre interacciones digitales, sociales y cognitivas. Tandil: Consejo Editorial de la UNCPBA, 2010. 175 p. 
SOLSONA, N. Los saberes científicos de las mujeres en el currículum. Qurriculum: Revista de Teoría, Investigación y Práctica Educativa, n. 28, p. 32-54, 2015.

TIBERGHIEN, A. Quelques éléments sur l'évolution de la recherche en didactique de la physique. Revue Française de Pédagogie, n. 72, p. 71-86, jul-sept. 1985.

TOUSSAINT, J. (Coord.) Didactique appliquée de la physique-chimie. Paris: Nathan, 1996.

VALDÉS, P. et al. Enseñanza de la física elemental. La Habana: Editorial Pueblo y Educación, 2002.

VIENNOT, L. Former en didactique, former sur le contenu ? Principes d'élaboration et éléments d'évaluation d'une formation en didactique de la physique en deuxième année d'IUFM. Didaskalia, n. 10, p. 75-96, 1997.

Agustín Adúriz-Bravo

CONICET/GEHyD - Grupo de Epistemología, Historia y Didáctica de las Ciencias Naturales, CeFIEC - Instituto de Investigaciones Centro de Formación e Investigación en Enseñanza de las Ciencias

Facultad de Ciencias Exactas y Naturales - Universidad de Buenos Aires.

Ciudad Autónoma de Buenos Aires - Argentina

(cc) BY-NC-ND Direito autoral e licença de uso: Este artigo está licenciado sob uma Licença Creative Commons.

\footnotetext{
${ }^{8}$ E-mail: aadurizbravo@cefiec.fcen.uba.ar
} 